\title{
Chromium-Doped ZnO Nanoparticles Synthesized by Co-Precipitation: Chromium Effects
}

\author{
Santi Septiani Sartiman, Nadia Febiana Djaja, Rosari Saleh
}

Departement Fisika, FMIPA-Universitas Indonesia, Depok, Indonesia.

Email: rosari.saleh@ui.ac.id, rosari.saleh@gmail.com

Received November $3^{\text {rd }}, 2012$; revised June $8^{\text {th }}, 2013$; accepted June $30^{\text {th }}, 2013$

Copyright (C) 2013 Santi Septiani Sartiman, et al. This is an open access article distributed under the Creative Commons Attribution License, which permits unrestricted use, distribution, and reproduction in any medium, provided the original work is properly cited.

\begin{abstract}
The influence of chromium doping on the physical properties of $\mathrm{ZnO}$ nanoparticles synthesized using a low temperature co-precipitation technique is presented. In particular, we have studied the correlation between the structural and the magnetic properties as a function of chromium concentrations. In order to investigate the magnetic properties, vibrating sample magnetometry and electron spin resonance were employed. X-ray diffraction, energy-dispersive X-ray spectroscopy, Fourier transform infrared spectroscopy and UV-Vis spectroscopy were used. X-ray diffraction patterns of all the samples showed peaks consistent with a hexagonal wurzite structure. The structure and composition analyses revealed that chromium is incorporated into the lattice structure, forming a solid solution instead of precipitates. All of the samples in this study exhibit ferromagnetic behavior. The implications of the effects of chromium are also discussed.
\end{abstract}

Keywords: Nanocrystalline ZnO Particles; Chromium; Room-Temperature-Ferromagnetic; ESR; Co-Precipitation

\section{Introduction}

The possibility to achieve both semiconducting and magnetic properties within a single material system by doping semiconducting materials with small concentrations of transition metals has spawned a new field of electronics known as spintronics [1-4]. The discovery of ferromagnetism at temperatures above $100 \mathrm{~K}$ in III-V-based ferromagnetic semiconductors such as Mn-doped GaAs has made it possible to fabricate spin injecting structures as well as structures for electrical or optical control of ferromagnetism [5-8]. However, most of the III-V-based ferromagnetic semiconductors have a highest Currie temperature $\left(\mathrm{T}_{\mathrm{C}}\right)$ of $110 \mathrm{~K}$, still far from room temperature, which is required for practical device applications [9-11].

Since Dietl et al. [12] predicted that p-type $\mathrm{ZnO}$ would be a promising candidate for high- $\mathrm{T}_{\mathrm{C}}$ ferromagnetic semiconductors, there has been considerable research and progress on transition metal-doped $\mathrm{ZnO}$ for the realization of a room temperature $T_{C}$. Thereafter, there has been considerable research and progress on transition metal doped $\mathrm{ZnO}$ for the realization of a $\mathrm{T}_{\mathrm{C}}$ at or above room temperature. Experimentally, room temperature ferromagnetism has been observed in Cr-, Mn-, Fe-, Co-, $\mathrm{Ni}$-, and $\mathrm{Cu}$-doped $\mathrm{ZnO}$ [13-18]. However, the results are inconsistent and controversial, both in experiments and in theory. This inconsistency suggests that room temperature ferromagnetism in transition metal-doped $\mathrm{ZnO}$ is highly sensitive to the synthesis procedures and conditions. Another suspicion is that ferromagnetism might be caused by a secondary phase, such as the doping elements or their oxides, rather than by the substitution of transition metal ions into $\mathrm{Zn}$ sites [19-21].

There are several theoretical explanations of the origin of room temperature ferromagnetism in transition metaldoped $\mathrm{ZnO}$. Dietl et al. [12] first predicted that a high $\mathrm{T}_{\mathrm{C}}$ for transition metal doped $\mathrm{ZnO}$ would require a large density of mobile holes to induce the ferromagnetic exchange interaction. However, a high concentration of hole is difficult to achieve due to the compensation of the wide band gap $\mathrm{ZnO}$. Additionally, such a theory cannot explain the RT ferromagnetism in n-type TM-doped $\mathrm{ZnO}$. Therefore, Sato and Katayama-Yoshida [22] suggested a theory based on a combination of the Korringa-KohnRostoker (KKR) method and the coherent potential approximation (CPA) to explain the origin of ferromagnetism in n-type TM-doped ZnO. Recently, Coey et al. [23] have argued that a carrier mediated exchange interaction cannot produce long-range magnetic order when 
the doping level of magnetic ions is only a few percent. They proposed a spin-split impurity band theory derived from the bound magnetic polarons (BMPs). In this model, the ferromagnetic exchange interaction is mediated by shallow donor electrons, which are treated in two-sublattice mean field approximation. The only remaining option to obtain a higher $\mathrm{T}_{\mathrm{C}}$ is to increase the donor electrons in the impurity band that will delocalize onto the TM ions.

Despite a number of reports available, confusion still persists on the existence and origin of room temperature ferromagnetism in transition metal doped $\mathrm{ZnO}$. Hence, an attempt was made to reinvestigate $\mathrm{Cr}$-doped $\mathrm{ZnO}$. For synthesis, a co-precipitation method was chosen because it is cost effective, it requires low temperatures for processing, and it has a higher degree of solubility. The powder samples were characterized with regard to their composition, structure and phase (EDX, XRD, and UV$\mathrm{Vis}$ ), and magnetic properties (VSM and ESR).

\section{Experimental}

The following starting materials were used without further purification: zinc (II) sulfate $\left(\mathrm{ZnSO}_{4} .7 \mathrm{H}_{2} \mathrm{O}, 99 \%\right.$, Merck)and chromium (III) chloride $\left(\mathrm{CrCl}_{3} \cdot 6 \mathrm{H}_{2} \mathrm{O}, 99 \%\right.$ Merck). To obtain the desired degree of doping of $\mathrm{Cr}$, $\mathrm{ZnSO}_{4} \cdot 7 \mathrm{H}_{2} \mathrm{O}$ was mixed in distilled water with

$\mathrm{CrCl}_{3} \cdot 6 \mathrm{H}_{2} \mathrm{O}$. This solutions were designated as solution A. Solution A was placed in an ultrasonic cleaner operating at $57 \mathrm{kHz}$ for $2 \mathrm{~h}$. Solution B was obtained by adding $44 \mathrm{mmol}$ of $\mathrm{NaOH}$ to $440 \mathrm{~mL}$ of de-ionized water. After sonication, solution A was mixed with a magnetic stirrer at room temperature, and solution B was added to solution A until a $\mathrm{pH}$ of 12 was reached. The resulting solution was magnetically stirred for $0.5 \mathrm{~h}$, and then it was allowed to stand at room temperature for $18 \mathrm{~h}$. Subsequently, the solution was centrifuged and washed several times with ethanol and distilled water to remove residual and unwanted impurities. The final product was dried in a vacuum oven at $200^{\circ} \mathrm{C}$ for $1 \mathrm{~h}$ to yieldCr-doped $\mathrm{ZnO}$ powder.

Elemental analyses of the samples were performed by energy dispersive X-ray (EDX) spectroscopy using a scanning electron microscope. Magnetic measurements were performed at room temperature using an Oxford Type $1.2 \mathrm{~T}$ vibrating sample magnetometer (VSM). The powder samples were tightly packed in a clear plastic drinking straw. Magnetization data were recorded as a function of an applied magnetic field, $0 \pm 1 \mathrm{~T}$. The data reported in this study were corrected because background signalswere introduced from the sample holder.

To evaluate the phase purity of the samples, X-ray diffraction (XRD) measurements were performed using a Philips PW 1710 and monochromatic Cu-Ka $(1=1.54060$
$\AA$ ) radiation operated at $40 \mathrm{kV}$ and $20 \mathrm{~mA}$ in the range of $10^{\circ}$ to $80^{\circ}$.The instrumental broadening, including the instrumental symmetry, was calibrated using a Si powder standard. The X-ray diffraction patterns were analyzed by means of the MAUD program using Rietveld whole profile fitting to determine the crystal structure and lattice parameters.

To study the electronic interactions near the optical band gap resulting from the addition of dopant atoms, diffuse reflectance UV-Vis measurements were performed using a Shimadzu UV-Vis spectrophotometer with an integrating sphere and a spectral reflectance standard over a wavelength range of $250-800 \mathrm{~nm}$. The diffuse reflectance, $R$, of the sample is related to the KubelkaMunk function, $F(R)$, according to the following equation:

$$
F(R)=(1-R)^{2} / 2 R
$$

The energy band gap of the samples was calculated from the diffuse reflectance spectra by plotting the $F(R)^{2}$ as a function of energy and extrapolating to $F(R)^{2}=0$.

To obtain information regarding the oxidation state and site occupancy of the $\mathrm{Cr}$ ions in the $\mathrm{ZnO}$ matrix, electron spin resonance (ESR) was performed using an $\mathrm{X}$-band JEOL JES-RE1X at room temperature and an $\mathrm{X}$-band spectrometer equipped with a $9.1 \mathrm{GHz}$ field modulation unit. The resonance was optimized for the modulation amplitude, receiver gain, time-constant and scan time. The amount of powder used in all measurements was the same. DPPH was used as the standard. The shape and area of the ESR spectra were analyzed using standard numerical methods.

\section{Results and Discussion}

\subsection{Chemical Analysis}

EDX measurement were used to determine the chemical composition of the $\mathrm{Cr}$-doped $\mathrm{ZnO}$ samples. Figure 1 shows EDX spectra of the $\mathrm{Cr}$-doped $\mathrm{ZnO}$ samples. The content of different elements in the sample can be observed in the spectra, confirming the incorporation of $\mathrm{Cr}$ atoms into the nanocrystalline $\mathrm{ZnO}$ particles. Calculating the area of the corresponding spectral $\mathrm{K}$ lines enabled quantitative characterization of the $\mathrm{Cr} / \mathrm{Zn}$ ratios.

The amounts of $\mathrm{Cr}$ in the nanocrystalline $\mathrm{ZnO}$ particles were determined to vary between 3 and 16 at.\%. The results were obtained by averaging four values from different regions of a sample. The inset of Figure 1 illustrates how the $\mathrm{Cr}$ incorporated into the nanocrystalline $\mathrm{ZnO}$ particles varied as a function of the initial cations ratio used in the synthesis. The EDX results indicate that the amounts of $\mathrm{Cr}$ incorporated in the samples were slightly lower than the amounts of $\mathrm{Cr}$ introduced in the synthesis. 


\subsection{Structural Characterization and Phase Evaluation}

Typical XRD patterns of $\mathrm{ZnO}$ nanoparticles doped with $\mathrm{Cr}$ at different concentrations are shown in Figure 2. A pure wurtzite, single phase structure of $\mathrm{ZnO}$ can be obtained for all samples doped with $\mathrm{Cr}$ concentrations up to 16 at.\%. No impurity peaks are detected, such as $\mathrm{Cr}_{2} \mathrm{O}_{3}$ and $\mathrm{ZnCr}_{2} \mathrm{O}_{4}$. This confirms that $\mathrm{Cr}$ dopant does not alter the crystal structure. However, the results of these data do not mean there is an absence of Cr clusters because we do not exclude the possibility of cluster formation below the detection limit of the X-ray diffractometer. TheXRD data were further analyzed by the Rietveld technique, using the program MAUD [24].

The evolution of lattice parameter $a$, and $c$ and the unit cell volume, $V$, with doping concentrations are plotted in

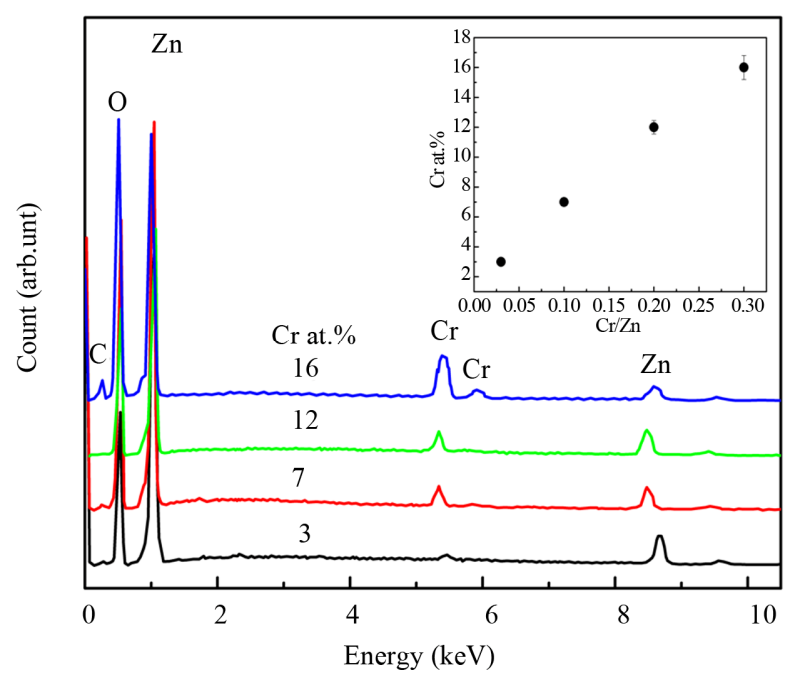

Figure 1. EDX spectra of Cr-doped nanocrystalline ZnO particles for various doping concentrations. For clarity, the spectra are shifted vertically.The inset shows the $\mathrm{Cr}$ incorporation in the nanoparticles as a function of the initial cation ratio in the starting solution.

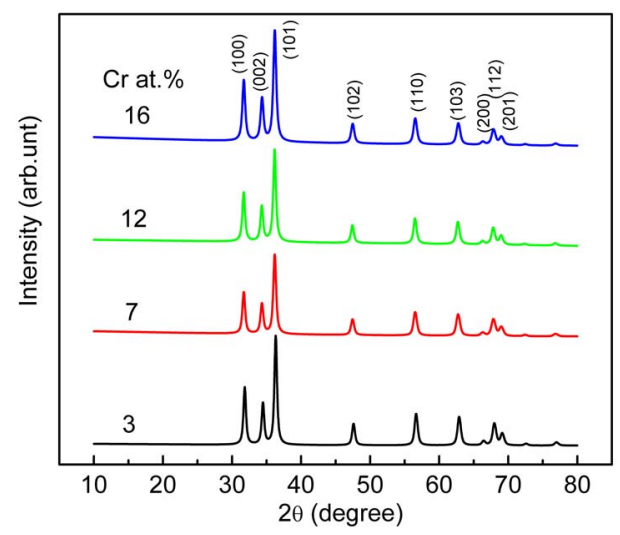

Figure 2. XRD patterns of Cr-doped nanocrystalline ZnO particles synthesized with different concentrations of $\mathrm{Cr}$.
Figure 3. For the Cr-doped samples, the lattice constant decreaseds with increasing $\mathrm{Cr}$ concentration. Such a decrease of the lattice parameters in $\mathrm{Cr}$-doped $\mathrm{ZnO}$ is quite obvious as the ionic radii of $\mathrm{Cr}$ ions are smaller than those of Zn.

\subsection{Microstrain and Stress Analysis}

It is known that the breadth of the XRD peak can be linked to the average crystallite size, microstrain and defects or dislocations. The average crystallite size using XRD measurements is not generally the same as the particle size due to powder aggregates. The average crystallite size as related to the line broadening can be calculated using Scherrer's equation, as given in Equation (2):

$$
\langle D\rangle=\frac{K}{(\beta \cos \theta)}
$$

where $\langle D>=$ volume weighted crystallite size, $K=$ shape factor (close to unity in our work, it was set to 0.9 ), $\lambda=$ wavelength of $\mathrm{Cu}-\mathrm{K}_{\mathrm{a}}, \quad b_{h k l}=\left[b_{h k l \text { measured }}^{2}-b_{\text {instrumental }}^{2}\right]^{1 / 2}$ $=$ instrumental corrected integral breadth of the reflection located at $2 \theta$, and $\theta=$ angle of reflection. The average crystallite size for the $\mathrm{Cr}$-doped $\mathrm{ZnO}$ calculated using

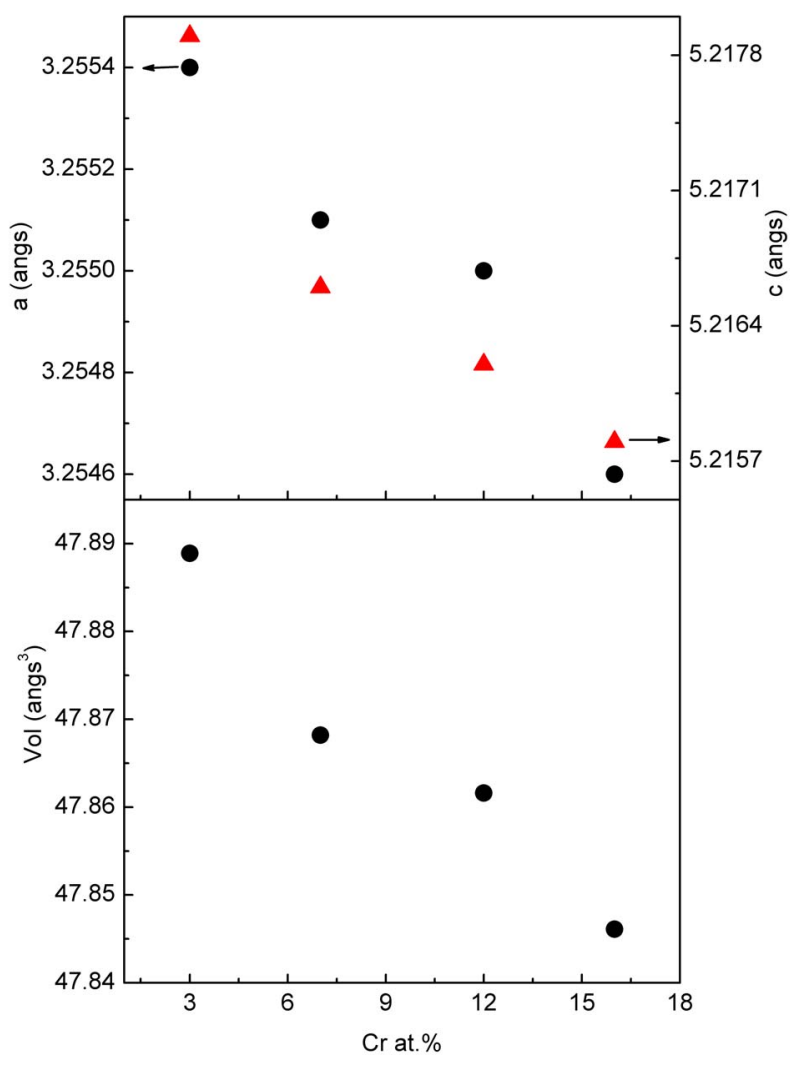

Figure 3. The lattice parameters a, $c$, and cell volume $V$ of Cr-doped nanocrystalline $\mathrm{ZnO}$ particles as a function of doping concentration. 
Scherrer's equation is presented in Table 1.

The strain arising from crystal imperfections and distortions, such as vacancies and lattice deformations, can also induce a broadening in the XRD profile. Therefore, the line broadening is a combination of crystallite size and strain. Although the separation of these two phenomena is not straightforward, the contribution of each type of broadening can be determined by constructing a modified form of William and Hall (W-H) plot [25], namely UDM. In this form the strain was assumed to be uniform in all crystallographic direction, thus the material is considered to be isotropic. Strain-induced broadening is calculated by the equation:

$$
\beta_{h k l}=4 \varepsilon \tan \theta
$$

where $\varepsilon=$ the lattice strain.

The UDM approach considers the case of both the domain effect and lattice deformation operating simultaneously, and their combined effects give the final line broadening, which is the sum of Equation (2) and Equation (3):

$$
\beta_{h k l}=\frac{K}{(D \cos \theta)}+4 \varepsilon \tan \theta
$$

Rearranging the equation, the following equation can be obtained:

$$
\beta_{h k l} \cos \theta=\frac{K}{\langle D\rangle}+4 \varepsilon \sin \theta
$$

By plotting the value of $4 \sin \theta$ along the $\mathrm{x}$-axis and $\beta_{h k l} \cos \theta$ along the y-axis, the lattice strain and the average crystallite size can be calculated through a linear fit. The UDM for Cr-doped $\mathrm{ZnO}$ are shown in Figure 4. It is clear that there is a decrease in the lattice strain with increasing doping concentrations. However, the UDM becomes less valid because the material properties vary with crystallographic directions. The other modified forms of the $\mathrm{WH}$ plot, that consider the anisotropic nature of the crystals are the uniform stress deformation model (USDM) and the uniform deformation energy density model (UDEDM). USDM and UDEDM give an idea of the stress-strain relation and the strain as a function of energy density, respectively. In USDM the anisotropic strain direction $\varepsilon_{h k l}$ can be related with the deformation stress using Young's modulus:

$$
\sigma_{h k l}=E_{h k l} \varepsilon_{h k l}
$$

where $E_{h k l}$ is Young's modulus in the direction perpendicular to the ( $h k l)$ plane and $\varepsilon_{h k l}$ is the anisotropic lattice strain. In this model the WH equation becomes:

$$
\beta_{h k l} \cos \theta=\frac{K}{\langle D\rangle}+\frac{4 \sigma \sin \theta}{E_{h k l}}
$$

The deformation stress can be estimated from the slope of the linear fit of the graph which is plotted between $\beta_{h k l} \cos \theta$ and $4 \sin \theta / E_{h k l}$, and the crystallite size $<\mathrm{D}>$ from the intercept. For samples with a hexagonal crystal phase, Young's modulus $E_{h k l}$ is related to:

$$
E_{h k l}=\frac{\left[h^{2}+\frac{(h+2 k)^{2}}{3}+\left(\frac{a l}{c}\right)^{2}\right]}{S_{11}\left(h^{2}+\frac{(h+2 k)^{2}}{3}\right)^{2}+S_{33}\left(\frac{a l}{c}\right)^{4}+\left(2 S_{13}+S_{44}\right)\left(h^{2}+\frac{(h+2 k)^{2}}{3}\right)\left(\frac{a l}{c}\right)^{2}}
$$

where $a$ and $c$ are lattice parameters and $S_{11}, S_{33}, S_{13}$ and $S_{44}$ are the elastic compliance of $\mathrm{ZnO}$ with values of 7.858 $\times 10^{-12},-2.206 \times 10^{-12}, 6.940 \times 10^{-12}$ and $23.57 \times 10^{-12}$ $\mathrm{m}^{2} \mathrm{~N}^{-1}$, respectively [26]. The USDMs for Cr-doped $\mathrm{ZnO}$ are also plotted in Figure 4. Using the linear fits of Figure

\begin{tabular}{|c|c|c|c|c|c|c|c|c|c|c|c|}
\hline \multirow{2}{*}{ Sample } & \multirow{2}{*}{ at. $\%$} & \multirow{2}{*}{$\begin{array}{c}\text { Debye Scherrer } \\
<\mathrm{D}>\mathrm{nm}\end{array}$} & \multicolumn{2}{|c|}{ UDM } & \multicolumn{3}{|c|}{ USDM } & \multicolumn{4}{|c|}{ UDEDM } \\
\hline & & & $<\mathrm{D}>\mathrm{nm}$ & e $\left(10^{-4}\right)$ & $<\mathrm{D}>\mathrm{nm}$ & $\sigma(\mathrm{MPa})$ & e $\left(10^{-4}\right)$ & $<\mathrm{D}>\mathrm{nm}$ & $\mathrm{u}\left(\mathrm{kJm}^{-3}\right)$ & $\sigma(\mathrm{MPa})$ & e $\left(10^{-4}\right)$ \\
\hline \multirow{4}{*}{$\mathrm{Cr}$ doped $\mathrm{ZnO}$} & 3 & 22 & 18 & 0.0007 & 17 & 71 & 0.0009 & 17 & 16 & 45 & 0.0005 \\
\hline & 7 & 20 & 15 & 0.0001 & 16 & 64 & 0.0008 & 16 & 13 & 41 & 0.0004 \\
\hline & 12 & 18 & 15 & 0.0001 & 15 & 11 & 0.0002 & 15 & 1 & 13 & 0.0001 \\
\hline & 16 & 17 & 14 & 0.0003 & 13 & 9 & 0.0001 & 14 & 4 & 23 & 0.0002 \\
\hline
\end{tabular}
4, the strain in anisotropic hexagonal crystals is calculated from the peak position, which is different from the USDM and UDM, their values are tabulated in Table 1.

Table 1. The average crystallite size, Young's modulus strain, stress, and anisotropic energy density of $\mathrm{Cr}$-doped na nocrystalline ZnO particles calculated using William-Hall approximation. 

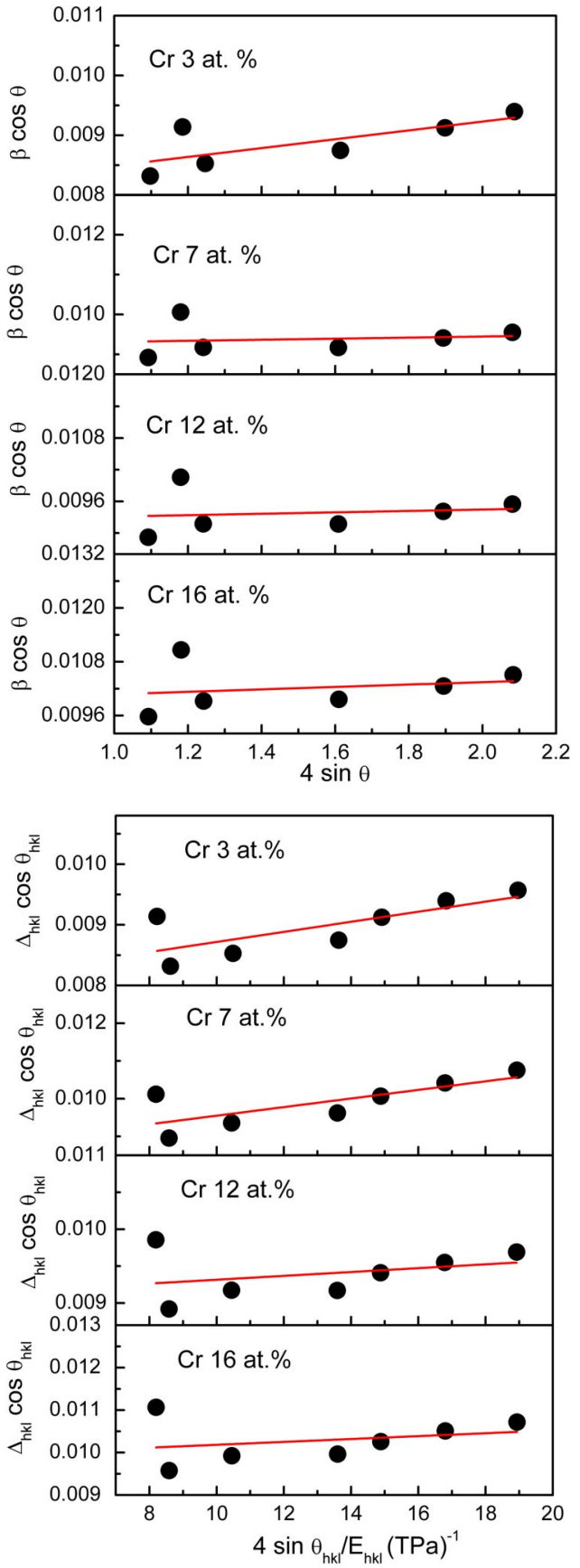

Figure 4. William-Hall plot for each doping concentration of Cr-doped nanocrystalline ZnO particles with UDM (up) and USDM (down) method.

In UDM, we have considered that the crystal is homogeneous isotropic, whereas in USDM, the assumption of homogeneity and isotropy is not fulfilled. Additionally, all of the constants proportionality associated with the stress-strain relation are no longer independent when the strain energy density, $u$, is considered. According to Hook's law the energy density is connected to the Young's modulus through the equation:

$$
u=\frac{\varepsilon^{2} E_{h k l}}{2}
$$

Therefore Equation (7) should be modified to the form:

$$
\beta_{h k l} \cos \theta=\frac{K}{\langle D\rangle}+4 \sin \theta\left(\frac{2 u}{E_{h k l}}\right)^{1 / 2}
$$

The anisotropic energy density, $u$, can be estimated from the slope of the curve $\beta_{h k l} \cos \theta$ as a function of $4 \sin \theta\left(2 / E_{h k l}\right)^{1 / 2}$. The average crystallite size can be obtained from the intercept and the strain from $s / E_{h k l}$. The variation of average crystallite size, strain lattice, stress and anisotropic energy density along with the $\mathrm{Cr}$ concentrations is shown in Table 1. The crystallite size calculated using Scherrer's formula differs slightly from that obtained from the UDM, USDM and UDEDM. It was observed that the strain and stress values decreased with decreasing average crystallite size as the dopant concentration was increased.

\subsection{Optical Properties}

To study the electronic interactions near the optical band gap region due to the presence of dopants diffuse-reflectance measurements were performed on the samples in the UV-Vis region at room temperature.

All spectra were obtained in the range of $200-800 \mathrm{~nm}$. Figure 5 shows the diffuse-reflectance spectra, R, as a function of the wavelength for the samples shown in Figure 2. The bandgap energy of the doped $\mathrm{ZnO}$ samples was calculated from the diffuse-reflectance spectra by plotting the square of the Kubelka-Munk function [27] $F(R)^{2}$ vs. the energy in electron volts. The linear part of the curve was extrapolated to $F(R)^{2}=0$ to calculate the direct bandgap energy. The inset of Figure 5 shows the bandgap as a function of the doping concentrations. The absorption edge shifts to lower energies/longer wavelengths. A similar shift in the absorption edge and band gap energy upon TM doping was reported in $\mathrm{Co}-$, $\mathrm{Ni}$-, and $\mathrm{Mn}$-doped $\mathrm{ZnO}$ nanoparticles [28-30] as well as in other TM-doped semiconductor nanoparticle systems [31-33].

Additionally to the reduction in the band gap energy, a decrease in the diffuse reflectance was also observed with increasing dopant concentration. A change in the band gap can be explained by the variation in the lattice parameters as a result of doping. The systematic variation of the diffuse-reflectance near the band edge for the doped samples in comparison with the undoped sample is a further confirmation of $\mathrm{Cr}$ ion incorporation in the $\mathrm{ZnO}$ lattice. The presence of $\mathrm{Cr}$ in the $\mathrm{ZnO}$ lattice is also consistent with our EDX results (Figure 1). Although the corresponding results were explained in terms of changes 


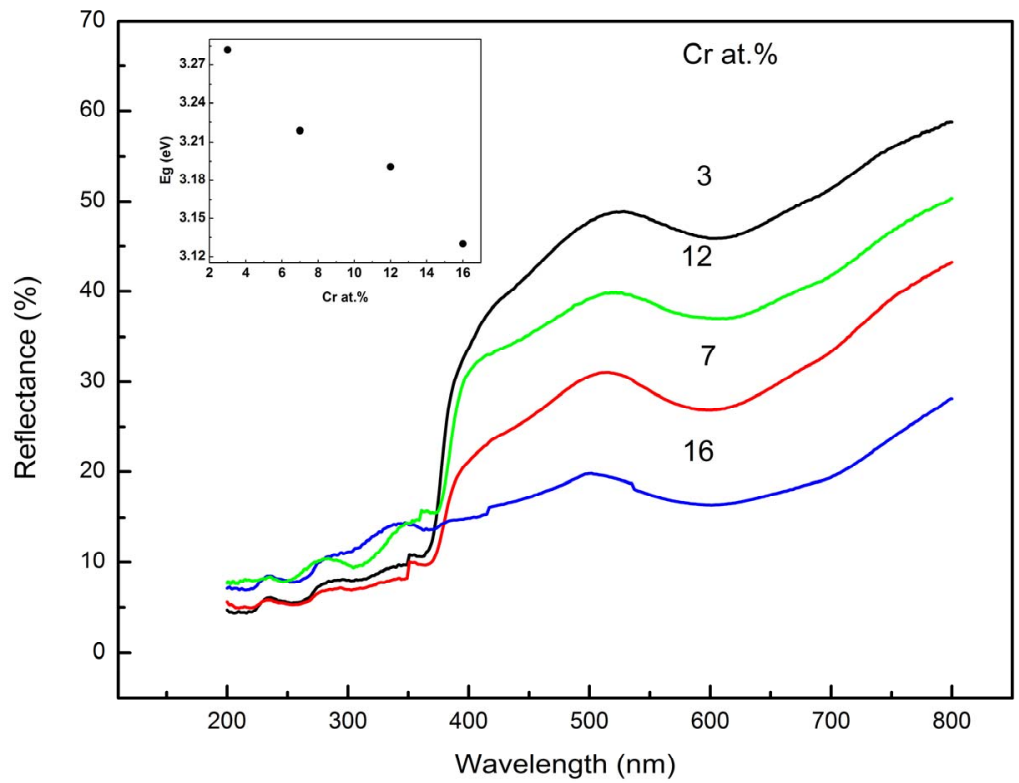

Figure 5. Diffuse-reflectance spectra of the Cr-doped nanocrystalline ZnO particles synthesized at various doping concentrations. The inset shows the correlated optical band gap of the $\mathrm{Cr}$-doped nanocrystalline $\mathrm{ZnO}$ particles as a function of the doping concentrations.

in the lattice parameters, other groups [34-36] argued that this factor is not likely to significantly influence the bandgap energy. They reported that the red shift in the bandgap energy of transitionmetal-doped II-VI semiconductors is attributed to the sp-d spin exchange interaction between the band electrons and the localized delectrons of the transition metal ion that substitutes the cation.

The exchange constant that constantly involves the s-like states decreases the energy of the bottom conduction band, the exchange factor involving the p-like states increases the energy of the top valence band by a constant factor that is independent of temperature.

\subsection{Electron Spin Resonance Study}

The ESR spectra for synthesized $\mathrm{Cr}$-doped $\mathrm{ZnO}$ at various composition observed at room temperature are shown in Figure 6. The spectra for all samples exhibited a very broad linewidth. The line width and intensity increased with increasing $\mathrm{Cr}$ content. All spectra had a symmetrical Lorentzian shape and have a similar lineshape. A stable electronic configuration of a free $\mathrm{Cr}$ atom is $3 d^{5} 4 s^{1}$, rather than $3 d^{4} 4 s^{2}$. As a dopant of the $\mathrm{ZnO}$ matrix, $\mathrm{Cr}$ can be incorporated in several ways [37]: 1) the electronic configuration of $\mathrm{Cr}$ can be $3 \mathrm{~d}^{4} 4 \mathrm{~s}^{2}$, with the two $4 \mathrm{~s}$ electrons incorporated in the tetrahedral bonding scheme, resulting in $\mathrm{Cr}^{2+}$ as an isoelectronic center; 2) a $3 \mathrm{~d}^{5} 4 \mathrm{~s}^{2}$ configuration can be formed by borrowing an electron from the valence band of the host with only one $4 \mathrm{~s}$ electron of $\mathrm{Cr}$ being incorporated in the tetrahedral bonding scheme, resulting in $\mathrm{Cr}^{+}$and causing the other 'borrowed' electron from the valence band to result in a negatively charged center to which the hole is bound; 3 ) $\mathrm{Cr}$ can also have a $3 \mathrm{~d}^{3}$ configuration, where the $\mathrm{Cr}^{3+}$ ion can incorporate one $4 \mathrm{~s}$ and one $3 \mathrm{~d}$ electron into the bonding scheme, while another $3 \mathrm{~d}$ is donated to the conduction band (making $\mathrm{Cr}$ a single donor), leaving the $\mathrm{Cr}$ center positively charged and able to bind the free electron. Considering the earlier works on ESR spectroscopy of II-VI compounds [38,39], one usually relates the ESR spectra detected in the Cr-doped II-VI semiconductors to the $\mathrm{Cr}^{2+}$ valance state. Therefore, it is also expected that the $\mathrm{Cr}^{2+}$ ion in the $\mathrm{Cr}$-doped $\mathrm{ZnO}$ will substitute for the $\mathrm{Zn}$ ion. However, our g-value and the peak-to-peak line width are in contradiction with the reported values of the ESR spectra of $\mathrm{Cr}^{2+}$ in II-VI compounds. Vallin et al. [40] showed that the $\mathrm{Cr}^{2+}$ ESR spectra in II-VI compounds have peak-to-peak line widths of $500 \mathrm{G}$ at $20 \mathrm{~K}$. Therefore, the expectation is that at room temperature, ESR signal of $\mathrm{Cr}^{2+}$ will be broader and unable to observed [41]. Additionally, a study of $\mathrm{Cr}$ doped GaAs [42] revealed that the $\mathrm{Cr}^{3+}$ valence state is predominant if the chromium concentration significantly exceeds the concentration of shallow donors in the crystal.

Therefore, we believed that our ESR signal arose from the $\mathrm{Cr}^{3+}$ centers instead of the $\mathrm{Cr}^{2+}$ ions. A similar result was also obtained by Krishnaiah et al. [43] in $\mathrm{ZnCrTe}$ crystals.All spectra lines were centered at approximately $550 \mathrm{G}$, corresponding to an effective g-value of 1.989 which is attributed to the typical value of $\mathrm{Cr}^{3+}$ ions $[42,45]$. All of the g-values of the spectrum observed in Cr-doped $\mathrm{ZnO}$ correspond not only to the $\mathrm{Cr}^{3+}$ ions 
observed in Cr-doped II-VI compounds, but also to an unpaired electron trapped at an oxygen vacancy site. It is possible that the broad line of the $\mathrm{Cr}^{3+}$ ions overlap with the resonance line of an unpaired electron trapped on an oxygen vacancy site. However, the deconvolution would be problematic because an accurate estimate of the intensity under the broad resonance line is difficult.

\subsection{Magnetic Characterization}

The magnetic properties of the $\mathrm{Cr}$-doped $\mathrm{ZnO}$ samples were investigated using VSM in the magnetic field range of 0 to $\pm 1 \mathrm{~T}$. The magnetization (M) versus the magnetic field $(\mathrm{H})$ measured at room temperature is illustrated in Figure 7. The magnetization is plotted as a function of magnetic field for different doping concentrations. All of the Cr-doped samples in this study exhibit ferromagnetic behavior.

An interesting feature is observed when we analyze the magnetization data of Cr-doped samples studied in this work. The 7, 12 and 16 at.\% Cr-doped samples showed the a remnant magnetizations of $0.35,0.35$, and $0.34\left(\times 10^{-2}\right) \mathrm{emu} / \mathrm{g}$ and coercive field of 482,322 and

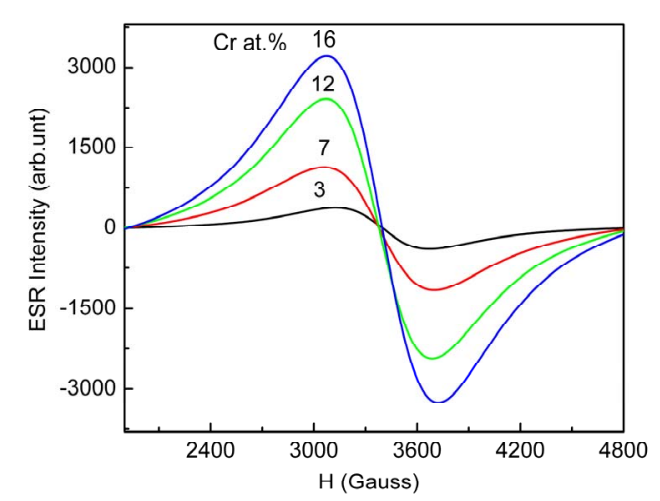

Figure 6. Experimental ESR spectra of the Cr-doped nanocrystalline $\mathrm{ZnO}$ particles prepared at various $\mathrm{Cr}$ concentrations.

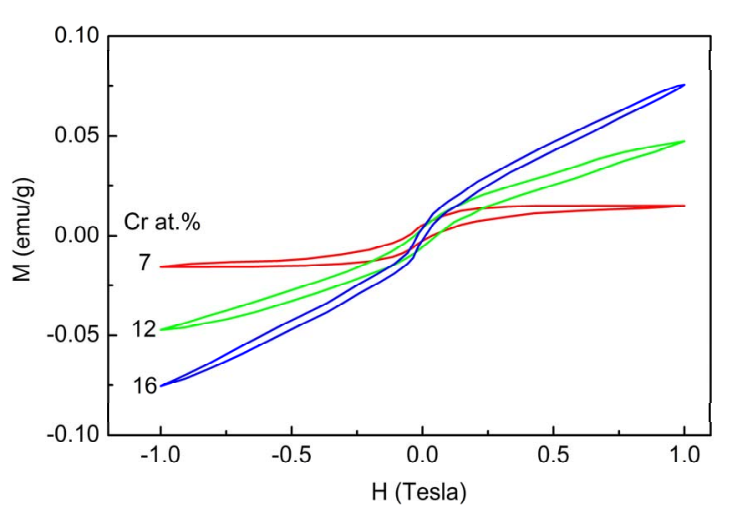

Figure 7. Room temperature $\mathrm{M}-\mathrm{H}$ curves for $\mathrm{Cr}$-doped nanocrystalline $\mathrm{ZnO}$ particles synthesized with various doping concentrations.
164 Oe, respectively. The magnetization increased for samples doped with higher $\mathrm{Cr}$ concentrations. The 7 at.\%, 12 at.\% and 16 at.\% Cr-doped samples possessed net moments of $0.060,0.077$ and $0.144 \mu_{\mathrm{B}} / \mathrm{Cr}$, respectively. The Cr-doped samples also had net moments far below the theoretical values. However, only a small fraction of $\mathrm{Cr}$ atoms contribute to ferromagnetism. The low magnetic moment values might be a result of the competition between the ferromagnetic and antiferromagnetic coupling that occurs at short distances between a pair of $\mathrm{Cr}$ ions.

Similar to $\mathrm{ZnO}$ doped with other transition metals, the secondary phase in our samples is thought to be the source of the spurious magnetic signal. In principle, as the $\mathrm{Cr}$ concentration increases, a number of antiferromagnetic phases may occur, such as $\mathrm{ZnCr}_{2} \mathrm{O}_{4}\left(\mathrm{~T}_{\mathrm{N}} 11 \mathrm{~K}\right)$, Cr metal clusters, $\mathrm{Cr}_{2} \mathrm{O}_{3}$ and $\mathrm{Cr}_{3} \mathrm{O}_{4}$ [46-48]. However, none of the phases can be detected via XRD. Even, if these phases are present in small quantities, none of them to ferromagnetism, except the $\mathrm{CrO}_{2}$ phase $\left(\mathrm{T}_{\mathrm{C}} \sim 386 \mathrm{~K}\right)$ [49]. Therefore, room temperature ferromagnetism in our $\mathrm{Cr}$ doped sample cannot be explained by the secondary phases. Xu et al. [50] observed ferromagnetism in Crdoped $\mathrm{ZnOnanorods.} \mathrm{They} \mathrm{observed} \mathrm{a} \mathrm{uniform} \mathrm{incorpo-}$ ration of $\mathrm{Cr}$ into $\mathrm{Zn}$ with no secondary phases and suggested that the combined effects of structural defects and exchange interactions of the $\mathrm{Cr}$ ions substituting for $\mathrm{Zn}$ in the $\mathrm{ZnO}$ matrix were responsible for the magnetization in their $\mathrm{Cr}$-doped $\mathrm{ZnO}$ samples.

The existence of compressive stress/and/or strain due to the oxygen vacancies may also be a factor that to influences the shift of the XRD peaks position. Defects are known to play a primary role in ferromagnetic ordering in transition metal-doped semiconductors such as $\mathrm{ZnO}$ $[23,46,51]$. An increasing number of studies have demonstrated that the formation of oxygen vacancies and zinc interstitial could lead to room temperature ferromagnetism or enhanced ferromagnetism [52-54]. According to the donor impurity band exchange model, the ferromagnetic-exchange between transition-metal ions and semiconductors is mediated by shallow donor electrons that form bound magnetic polarons which overlap to create a spin-split impurity band.

With regard to the origin of the ferromagnetism observed in our samples and considering the above measurements, we conclude that ferromagnetism in our $\mathrm{Cr}$ doped samples is an intrinsic property of $\mathrm{Cr}$-doped $\mathrm{ZnO}$. Our data support the hypothesis that the magnetic behavior observed is related to the presence of intrinsic defects. This can be understood via the BMP model discussed above.

\section{Summary}

In summary, a series of $\mathrm{Cr}$-doped $\mathrm{ZnO}$ nanoparticle samples with different doping concentrations were success- 
fully synthesized and characterized by EDX, XRD, UV-Vis, ESR and VSM. The XRD patterns suggested the formation of a wurtzite structure in theCr-doped $\mathrm{ZnO}$. The XRD results also indicated that $\mathrm{Cr}$-ions were incurporated into the $\mathrm{ZnO}$ matrix. The XRD line broadening due to the combination of the coherent scattering of Xrays from a particular lattice plane and the random displacement of atoms from their original positions, which generates strain was analyzed by Scherrer's formula and by a modified form of W-H analysis. The three modified $\mathrm{W}-\mathrm{H}$ analyses were helpful in approximating the strain, stress and energy density values. The results show that the strain, stress and energy density values decreased with increasing doping concentrations.

The magnetic measurements show that the Cr-doped $\mathrm{ZnO}$ samples are ferromagnetic in nature with a welldefined hysteresis at room temperature and the coercive field $\left(\mathrm{H}_{\mathrm{C}}\right)$ and the remnant magnetization increase with increasing doping concentrations. The analysis shows that the room temperature ferromagnetic in Cr-doped $\mathrm{ZnO}$ might be explained by BMP model.

\section{REFERENCES}

[1] G. A. Prinz, "Magnetoelectronics," Science, Vol. 282, No. 5394, 1998, pp. 1660-1663. doi:10.1126/science. 282.5394 .1660

[2] A. Wolf, D. D. Awschalom, R. A. Buhrman, J. M. Daughton, S. von Molnár, M. L. Roukes, A. Y. Chtchelkanova and D. M. Treger, "Spintronics: A Spin-Based Electronics Vision for the Future," Science, Vol. 294, No. 5546, 2001, pp. 1488-1495. doi:10.1126/science. 1065389

[3] S. J. Pearton, C. R. Abernathy, M. E. Overberg, G. T. Thaler, D. P. Norton, N. Theodoropoulou, A. F. Hebard, Y. D. Park, F. Ren, J. Kim and L. A. Boatner, "Wide Band Gap Ferromagnetic Semiconductors and Oxides," Journal of Applied Physics, Vol. 93, No. 1, 2003, 13 p. doi:10.1063/1.1517164

[4] K. Ueda, H. Tabata and T. Kawai, "Magnetic and Electric Properties of Transition-Metal-Doped ZnO Films," Applied Physics Letters, Vol. 79, No. 7, 2001, pp. 988-990. doi:10.1063/1.1384478

[5] H. Ohno, "Making Nonmagnetic Semiconductors Ferromagnetic," Science, Vol. 281, No. 5379, 1998, pp. 951956. doi:10.1126/science.281.5379.951

[6] H. Ohno, H. Munekata, T. Penney, S. Von Molnár and L. L. Chang, "Magnetotransport Properties of p-Type (In, Mn)As Diluted Magnetic III-V Semiconductors," Physical Review Letters, Vol. 68, No. 17, 1992, pp. 2664-2667. doi:10.1103/PhysRevLett.68.2664

[7] H. Ohno, A. Shen, F. Matsukura, A. Oiwa, A. Endo, S. Katsumoto and Y. Iye, "(Ga,Mn)As: A New Diluted Magnetic Semiconductor Based on GaAs," Applied Physics Letters, Vol. 69, No. 3, 1996, pp. 363-365. doi:10.1063/1.118061

[8] F. Matsukura, H. Ohno, A. Shen and Y. Sugawara, "Transport Properties and Origin of Ferromagnetism in (Ga,Mn)
As," Physical Review B, Vol. 57, No. 4, 1998, pp. R2037R2040. doi:10.1103/PhysRevB.57.R2037

[9] Y. Matsumoto, M. Murakami, T. Shono, T. Hasegawa, T. Fukumura, M. Kawasaki, P. Ahmet, T. Chikyow, S. Koshihara and H. Koinuma, "Room-Temperature Ferromagnetic in Transparent Transition Metal-Doped Titanium Dioxide," Science, Vol. 291, No. 5505, 2001, pp. 854-856. doi:10.1126/science.1056186

[10] T. Wakano, N. Fujimura, Y. Morinaga, N. Abe, A. Ashida and T. Ito, "Magnetic and Magneto-Transport Properties of ZnO:Ni Films," Physica E: Low-Dimensional Systems and Nanostructures, Vol. 10, No. 1-3, 2001, pp. 260-264. doi:10.1016/S1386-9477(01)00095-9

[11] P. Sharma, A. Gupta, K. V. Rao, F. J. Owens, R. Sharma, R. Ahuja, J. M. Osorio, B. Johansson and G. A. Gehring, "Ferromagnetism above Room Temperature in Bulk and Transparent Thin Films of Mn-Doped ZnO," Nature Matter, Vol. 2 , No. 10, 2003, pp. 673-677. doi:10.1038/nmat984

[12] T. Dietl, H. Ohno, F. Matsukura, J. Cibert and D. Ferrand, "Zener Model Description of Ferromagnetism in ZincBlende Magnetic Semiconductors," Science, Vol. 287, No. 5455, 2000, pp. 1019-1022. doi:10.1126/science.287.5455.1019

[13] R. Saleh, S. P. Prakoso and A. Fishli, "The Influence of Fe Doping on the Structural, Magnetic and Optical Properties of Nanocrystalline Zno Particles," Journal of Magnetism and Magnetic Materials, Vol. 324, No. 5, 2012, pp. 665-670. doi:10.1016/j.jmmm.2011.07.059

[14] G. Glaspell, P. Dutta and A. A Manivanna, "A RoomTemperature and Microwave Synthesis of M-Doped ZnO ( $\mathrm{M}=\mathrm{Co}, \mathrm{Cr}, \mathrm{Fe}, \mathrm{Mn} \& \mathrm{Ni})$, , Journal of Cluster Science, Vol. 16, No. 4, 2005, pp. 523-536. doi:10.1007/s10876-005-0024-y

[15] O. D. Jayakumar, H. G. Salunke, R.M. Kadam, M. Mohapatra, G. Yaswant and S. K. Kulshreshtha, "Magnetism in Mn-doped ZnO Nanoparticles Prepared by A Co-Precipitation Method," Nanotechnology, Vol. 17, No. 5, 2006, p. 1278. doi:10.1088/0957-4484/17/5/020

[16] R. Elilaressi and G. Chandrasekaran, "Structural, Optical, and Magnetic Properties of Nanoparticles of $\mathrm{ZnO}: \mathrm{Ni}-$ DMS Prepared by Sol-Gel Method," Materials Chemistry Physics, Vol. 123, No. 2-3, 2010, pp. 450-455. doi:10.1016/j.matchemphys.2010.04.039

[17] D. B. Buchholz, R. P. H. Chang, J.-Y. Song and J. B. Ketterson, "Room-Temperature Ferromagnetism in $\mathrm{Cu}$ Doped ZnO Thin Films," Applied Physics Letters, Vol. 87, No. 8, 2005, Article ID: 082504. doi:10.1063/1.2032588

[18] L.-H. Ye, A. J. Freeman and B. Delley, "Half-Metallic Ferromagnetism in $\mathrm{Cu}$-Doped $\mathrm{ZnO}$ : Density Functional Calculations," Physical Review B, Vol. 73, No. 3, 2006, Article ID: 033203. doi:10.110x3/PhysRevB.73.033203

[19] K. P. Bhatti, S. Chaudhary, D. K. Pandya and S. C. Kashyap, "Intrinsic and Extrinsic Origin of Room Temperature Ferromagnetism in $\mathrm{ZnO}$ :Co (5 at. \%)," Journal of Applied Physics, Vol. 101, No. 10, 2007, Article ID: 103919. doi:10.1063/1.2740343

[20] J. Zhang, R. Skomski and D. J. Sellmyer, "Sample Preparation and Annealing Effects on the Ferromagnetism 
in Mn-Doped ZnO," Journal of Applied Physics, Vol. 97, No. 10, 2005, Article ID: 10D303.

[21] D. P. Norton, M. E. Overberg, S. J. Pearton, K. Pruessner, J. D. Budai, L. A. Boatner, M. F. Chisholm, J. S. Lee, Z. G. Khim, Y. D. Park and R. G. Wilson, "Ferromagnetism in Cobalt-Implanted ZnO," Applied Physics Letters, Vol. 83, No. 26, 2003, p. 5488. doi:10.1063/1.1637719

[22] K. Sato and H. Katayama-Yoshida, "Ferromagnetism in a Transition Metal Atom Doped ZnO," Physica E: LowDimensional Systems and Nanostructures, Vol. 10, No.1, 2001, pp. 251-255. doi:10.1016/S1386-9477(01)00093-5

[23] J. M. Coey, M. Venkatesan and C. B. Fitzgerald, "Donor Impurity Band Exchange in Dilute Ferromagnetic Oxides," Nature Matter, Vol. 4, No. 2, 2005, pp. 173-179. doi:10.1038/nmat1310

[24] L. Lutterotti, "MAUD (Material Analysis Using Diffraction) version 2.33," 2010.

http://www.ing.unitn.it/ luttero/maud

[25] G. K. Williamson and W. H. Hall, "X-Ray Line Broadening from Filled Aluminium and Wolfram," Acta Metallurgica, Vol. 1, No. 1, 1953, pp. 22-31. doi:10.1016/0001-6160(53)90006-6

[26] J. F. Nye, "Physical Properties of Crystals: Their Representation by Tensors and Matrices," Oxford University Press, Oxford, New York, 1985.

[27] B. Hapke, "Theory of Reflectance and Emittance Spectroscopy," University Press, Cambridge, 1993.

[28] P. Koidl, "Optical Absorption of $\mathrm{Co}^{2+}$ in $\mathrm{ZnO}$," Physical Review B, Vol. 15, No. 5, 1977, pp. 2493-2499. doi:10.1103/PhysRevB.15.2493

[29] J. Anghel, A. Thurber, D. A. Tenne, C. B. Hanna and A. Punnoose, "Correlation Between Saturation Magnetization, Bandgap, and Lattice Volume of Transition Metal $(\mathrm{M}=\mathrm{Cr}, \mathrm{Mn}, \mathrm{Fe}, \mathrm{Co}$, or Ni) doped Zn1-XMXO Nanoparticles," Journal of Applied Physics, Vol. 107, No. 9, 2010, Article ID: 09E314. doi:10.1063/1.3360189

[30] Z. H. Wang, D. Y. Geng and Z. D. Zhang, "Room-Temperature Ferromagnetism and Optical Properties of Zn1XMnXO Nanoparticles," Solid State Communication, Vol. 149, No. 17-18, 2009, pp. 682-684. doi:10.1016/j.ssc.2009.02.016

[31] M. Bouloudenine, N. Viart, S. Colis and A. Dinia, "Bulk Zn1-XCoXO MAGNETIC Semiconductors Prepared by Hydrothermal Technique," Chemical Physics Letters, Vol. 397, No. 1-3, 2004, pp. 73-76. doi:10.1016/j.cplett.2004.08.064

[32] K. J. Kim and Y. R. Park, "Spectroscopic Ellipsometry Study of Optical Transitions in Zn1-XCoXO Alloys," Applied Physics Letters, Vol. 81, No. 8, 2002, p. 1420. doi:10.1063/1.1501765

[33] A. J. Hays, A. Punnoose, R. Baldner, M. H. Engelhard, J. Peloquin, K. M. Reddy, "Relationship between the Structural and Magnetic Properties of Co-Doped $\mathrm{SnO}_{2}$ Nano-particles," Physical Review B, Vol. 72, No. 7, 2005, Article ID: 075203. doi:10.1103/PhysRevB.72.075203

[34] Z. W. Zhao, B. K. Tay, J. S. Chen, J. F. Hu, B. C. Lim and G. P. Li, "Large Magnetic Moment Observed in Co-Doped $\mathrm{ZnO}$ Nanoclusters-Assembled Thin Films at
Room Temperature," Applied Physics Letters, Vol. 90 No. 15, 2007, Article ID: 152502. doi:10.1063/1.2721140

[35] A. Parra-Palomino, O. Perales-Perez, R. Singhal, M. Tomar, J. Hwang, P. M. Voyles, "Structural, Optical, and Magnetic Characterization of Monodisperse Fe-Doped ZnO Nanocrystals," Journal of Applied Physics, Vol. 103, No. 7, 2008, Article ID: 07D121. doi:10.1063/1.2834705

[36] Z. H. Wang, D. Y. Geng, and Z. D. Zhang, "Room-Temperature Ferromagnetism and Optical Properties of $\mathrm{Zn}_{1-\mathrm{X}}$ $\mathrm{Mn}_{\mathrm{X}} \mathrm{O}$ Nanoparticles," Solid State Communications, Vol. 149, No. 17-18, 2009, pp. 682-684. doi:10.1016/j.ssc.2009.02.016

[37] X. Lu, S. Tsoi, I. Miotkowski, S. Rodriguez, H. Alawadhi and A. K. Ramdas, "Raman Electron Paramagnetic Resonance in $\mathrm{Zn}_{1-\mathrm{x}} \mathrm{Cr}_{\mathrm{x}}$ Te and $\mathrm{Cd}_{1-\mathrm{x}} \mathrm{Cr}_{\mathrm{x}}$ Te," Physical Review B, Vol. 75, No. 15, 2007, Article ID: 155206. doi:10.1103/PhysRevB.75.155206

[38] J. T. Vallin and G. D. Watkins, "The Spin Hamiltonian for $\mathrm{Cr}^{2+}$ in CdS," Physics Letters A, Vol. 37, No. 4, 1971, pp. 297-298. doi:10.1016/0375-9601(71)90678-5

[39] T. L. Estle and W. C. Holton, "Electron-ParamagneticResonance Investigation of the Superhyperfine Structure of Iron-Group Impurities in II-VI Compounds," Physical Review, Vol. 150, No. 1, 1966, pp. 159-167. doi:10.1103/PhysRev.150.159

[40] J. T. Vallin and G. D. Watkins, "EPR of $\mathrm{Cr}^{2+}$ in II-VI Lattices," Physical Review B, Vol. 9, No. 5, 1974, pp. 2051-2072. doi:10.1103/PhysRevB.9.2051

[41] M. E. J. Boonman, W. Mac, A. Twardowski, A. Wittlin, P. J. M. van Bentum, J. C. Maan and M. Demianiuk, "High-Magnetic-Field EPR of Cr-Based Diluted Magnetic Semiconductors," Physical Review B, Vol. 61, No. 8, 2000, pp. 5358-5368. doi:10.1103/PhysRevB.61.5358

[42] J. J. Kerbs and G. H. Strauss, "EPR of $\mathrm{Cr}\left(3 \mathrm{~d}^{3}\right)$ in GaAs-Evidence for Strong Jahn-Teller Effects," Physical Review B, Vol. 15, No. 1, 1977, pp. 17-22. doi:10.1103/PhysRevB.15.17

[43] G. Krishnaiah, N. MadhusudhanaRao, D. R. Reddy, B. K. Reddy and P. S. Reddy, "Growth and Structural Properties of $\mathrm{Zn}_{1-\mathrm{x}} \mathrm{Cr}_{\mathrm{x}}$ Te Crystals," Journal of Crystal Growth, Vol. 310, No. 1, 2008, pp. 26-30. doi:10.1016/j.jcrysgro.2007.10.013

[44] A. Franco Jr. and R. C. Santana, "Electron Paramagnetic Resonance (EPR) of Antiferromagnetic Nanoparticles of $\mathrm{La}_{1-\mathrm{x}} \mathrm{Sr}_{\mathrm{x}} \mathrm{CrO}_{3}(0.000 \leqslant \mathrm{x} \leqslant 0.020)$ Synthesized by Combustion Reaction," Materials Chemistry and Physics, Vol. 120, No. 1, 2010, pp. 225-228. doi:10.1016/j.matchemphys.2009.10.052

[45] G. Krishnaiah, N. MadhusudhanaRao, B. K. Reddy, D. R. Reddy, T. M. Babu, S. Sambasivam and P. S. Reddy, "EPR and Magnetic Properties of Vapour Phase Grown $\mathrm{Zn}_{1-\mathrm{x}} \mathrm{Cr}_{\mathrm{x}}$ Te Crystals," Physics Letters A, Vol. 372, No. 42, 2008, pp. 6429-6433. doi:10.1016/j.physleta.2008.08.039

[46] H. Liu, X. Zhang, L. Li, Y. X. Wang, K. H. Gao, Z. Q. Li, R. K. Zheng, S. P. Ringer, B. Zhang and X. X. Zhang, "Role of Point Defects in Room-Temperature Ferromagnetism of Cr-Doped ZnO," Applied Physics Letters, Vol. 91, No. 7, 2007, Article ID: 072511. 


$$
\text { doi:10.1063/1.2772176 }
$$

[47] X. H. Chen, H. T. Zhang, C. H. Wang, X. G. Luo and P. H. Li, "Effect of Particle Size on Magnetic Properties of Zinc Chromite Synthesized by Sol-Gel Method," Applied Physics Letters, Vol. 81, No. 23, 2002, p. 4419. doi: $10.1063 / 1.1526921$

[48] B. K. Roberts, A. B. Pakhomov and K. M. Krishnan, "Effect of Hydrogen Codoping on Magnetic Ordering and Conductivity in Cr:ZnO Thin Films," Journal of Applied Physics, Vol. 103, No. 7, 2008, Article ID: 07D133.

[49] R. Bhargava, P. K Sharma, A. K Chawla, S. Kumar, R. Chandra, A. C Pandey and N. Kumar, "Variation in Structural, Optical and Magnetic Properties of $\mathrm{Zn}_{1-\mathrm{X}} \mathrm{Cr}_{\mathrm{X}} \mathrm{O}$ $(\mathrm{x}=0.0,0.10,0.15$, and 0.20$)$ Nanoparticles: Role of Dopant Concentration on Non-Saturation of Magnetization," Materials Chemistry and Physics, Vol. 125, No. 3, 2011, pp. 664-671. doi:10.1016/j.matchemphys.2010.09.075

[50] C. Xu, K. Yang, Y. Liu, L. Huang, H. Lee, J. Cho and H. Wang, "Buckling and Ferromagnetism of Aligned CrDoped ZnO Nanorods," The Journal of Physical Chemis- try C, Vol. 112, No. 49, 2008, pp. 19236-19241. doi:10.1021/jp806164b

[51] C. Song, K. W. Geng, F. Zeng, X. B. Wang, Y. X. Shen, F. Pan, Y. N. Xie, T. Liu, H. T. Zhou and Z. Fan, "Giant Magnetic Moment in an Anomalous Ferromagnetic Insulator: Co-Doped ZnO," Physical Review B, Vol. 73, No. 2, 2006, Article ID: 024405. doi:10.1103/PhysRevB.73.024405

[52] J. L. MacManus-Driscoll, N. Khare, Y. Liu and M. E. Vickers, "Structural Evidence for Zn Intersititials in Ferromagnetic $\mathrm{Zn}_{1-\mathrm{x}} \mathrm{Co}_{\mathrm{x}} \mathrm{O}$ Films," Advanced Materials, Vol. 19, No. 19, 2007, pp. 2925-2929.

[53] H. S. Hsu, J. C. A. Huang, S. F. Chen and C. P. Liu, "Role of Grain Boundary and Grain Defects on Ferromagnetism in Co:ZnO Films," Applied Physical Letters, Vol. 90, No. 10, 2007, Article ID: 102506. doi:10.1063/1.2711763

[54] J. M. D. Coey, "Dilute Magnetic Oxide," Current Opinion in Solid State and Materials Science, Vol. 10, No. 2, 2006, pp. 83-92. doi:10.1016/j.cossms.2006.12.002. 